\title{
Deshidratación osmótica en frutos de carambola (Averrhoa carambola) en Pucallpa
}

\section{Dehydration in carambola fruits (Averrhoa carambola) in Pucallpa}

\author{
Erika Lizbeth Garay Torres ${ }^{1}$, Edgardo García Saavedra ${ }^{1}$, Caleb Leandro Laguna ${ }^{2}$, Dina Pari Quispe ${ }^{1}$, Ynés \\ del Carmen Tavera Arévalo ${ }^{1}$ y Manuel Mamani Flores ${ }^{1}$
}

\begin{abstract}
${ }^{1}$ Universidad Nacional de Ucayali - Perú. Carretera Federico Basadre Km 5.5, Callería. Ucayali, Perú. Email: Erika_garay@unu.edu.pe ORCID: https://orcid.org/0000-0001-6299-7692

${ }^{1}$ Universidad Nacional de Ucayali - Perú. Carretera Federico Basadre Km 5.5, Callería. Ucayali, Perú. Email: garcia_se73@hotmail.com. ORCID: https://orcid.org/0000-0001-6299-7692

${ }^{1}$ Universidad Nacional de Ucayali - Perú. Carretera Federico Basadre Km 5.5, Callería. Ucayali, Perú. Email: dina_pari@unu.edu.pe. ORCID: https://orcid.org/0000-0002-1493-79209

${ }^{1}$ Universidad Nacional de Ucayali - Perú. Carretera Federico Basadre Km 5.5, Callería. Ucayali, Perú. Email: ines_tavera@unu.edu.pe ORCID: https://orcid.org/0000-0001-7133-4030

${ }^{1}$ Universidad Nacional de Ucayali - Perú. Carretera Federico Basadre Km 5.5, Callería. Ucayali, Perú. Email: katmt23@gmail.com. ORCID: https://orcid.org/0000-0002-0431-1813

${ }^{2}$ Universidad Nacional Intercultural de la Amazonía - Perú. Carretera a San José km. 0.63, Pucallpa, Perú. Email: caleb_leandro@unia.edu.pe, Ucayali, Perú. ORCID: https://orcid.org/0000-0002-6168-1079
\end{abstract}

\section{Resumen}

Se evaluó el comportamiento de la madurez, la concentración del agente osmótico y temperatura en la cinética de osmodeshidratado de carambola con miel de caña de azúcar, para lo cual, los frutos fueron lavados, desinfectados, se les retiraron las aristas y la cáscara, dejando la pulpa, para cortarlos en rodajas transversales, los cuales fueron sumergidos en los tratamientos de miel de caña (pasteurizada a $50^{\circ} \mathrm{C}$ y diluidas a 40,50 y $60^{\circ}$ Brix), aplicando temperaturas de deshidratación de 40 y $50{ }^{\circ} \mathrm{C}$, quitándoles el exceso de miel de caña, y sometidos a $85^{\circ} \mathrm{C}$ por 5 horas en una estufa y posteriormente enfriadas y colocadas en envases con tapa, almacenándolos a temperatura ambiente. El análisis sensorial se realizó con la participación de treinta panelistas semi entrenados. Se aplicó un Diseño de Bloque Completamente al azar (DBC) con arreglo factorial $2 \times 3 \times 2$, y una prueba de medias de Tukey al 0,05\% de significancia, concluyéndose que, la carambola osmodeshidratada muestra una disminución en la acidez, y un aumento significativo en el $\mathrm{pH},{ }^{\circ} \mathrm{Brix}$, cenizas y fibra, asimismo, los frutos pintones muestran mayor ganancia de sólidos solubles, y la ganancia de sólidos solubles se incrementa al incrementarse la concentración de la solución osmótica empleada, y la ganancia de sólidos solubles es directamente proporcional a la temperatura de proceso. Todos los tratamientos muestran efectividad del osmodeshidratado, finalmente, los tratamientos estudiados no ejercen efecto significativo sobre el agrado general de la carambola osmodeshidratada y los resultados del análisis microbiológico, muestran un recuento inferior al $50 \mathrm{ufc/g}$ para mohos y levaduras.

Palabras clave: carambola, deshidratación osmótica, ganancia de sólidos, pérdida de masa, miel de caña. 


\begin{abstract}
The maturity behavior, the concentration of the osmotic agent and temperature in the kinetics of carambola osmodehydrated with sugar cane honey were evaluated, for which, the fruits were washed, disinfected, the edges and the peel were removed, leaving the pulp, to cut them into transverse slices, which were immersed in the cane honey treatments (pasteurized at $50^{\circ} \mathrm{C}$ and diluted at 40,50 and $60^{\circ}$ Brix), applying dehydration temperatures of 40 and $50^{\circ} \mathrm{C}$, removing excess cane honey, and subjected to $85^{\circ} \mathrm{C}$ for 5 hours in an oven and then cooled and placed in containers with lids, storing them at room temperature. Sensory analysis was performed with the participation of thirty semitrained panelists. A Completely randomized Block Design (DBC) with a $2 \times 3 \times 2$ factorial arrangement, and a Tukey mean test at $0.05 \%$ significance, were applied, concluding that osmodehydrated carambola shows a decrease in acidity, and a significant increase in $\mathrm{pH},{ }^{\circ}$ Brix, ash and fiber, likewise, the Painted fruits show a greater gain in soluble solids, and the gain in soluble solids increases with increasing concentration of the osmotic solution used, and the gain in soluble solids it is directly proportional to the process temperature. All the treatments show the effectiveness of the osmodehydrated, finally, the studied treatments do not exert a significant effect on the general liking of the starch osmodehydrated and the results of the microbiological analysis show a count lower than $50 \mathrm{cfu} / \mathrm{g}$ for molds and yeasts.
\end{abstract}

Keywords: carambola, osmotic dehydration, solids gain, mass loss, cane hone.

\section{Introducción}

La carambola es una fruta exótica con un aporte nutrimental importante que se refleja por su elevada concentración de compuestos bioactivos como polifenoles, vitaminas $\mathrm{A}, \mathrm{C} \mathrm{y}$ minerales; sin embargo, debido a su alto contenido de agua es un producto altamente perecedero (Castillo-Zamudio et al., 2018), razón por la cual existe la necesidad de conservar las frutas, para poder alargar su vida útil, facilitar el almacenaje, transporte y manipulación de las mismas. Para ello se desarrollan diferentes métodos de conservación como la liofilización, la cual permite obtener productos deshidratados que conserven su calidad nutritiva, sensorial y funcional (Pucahuaylla \& Valdivieso, 2018).

Existiendo así la deshidratación que es el proceso de extracción del agua que contiene mediante la circulación de aire caliente, lo que detiene el crecimiento de enzimas y microorganismos que lo deterioran (Ochoa Icaza, 2019); así se tiene se tiene que el proceso de la deshidratación osmótica, es un proceso de transferencia de masa, disminución de humedad y el aumento de sólidos que dependen de la fuerza del flujo y la resistencia del producto, las cuales son afectados por: el tipo de materia prima, tiempo, temperatura, velocidad de agitación, peso molecular, tipo y 
concentración de la solución osmótica, siendo reforzado con el secado convectivo para mejorar las características físico-químicas de los frutos y/o hortalizas a procesar (Meneses, 2018).

La deshidratación osmótica, consiste en la inmersión de un alimento sólido, en soluciones acuosas de sacarosa o sal de alta concentración en solutos (hipertónica) a un tiempo y temperaturas específicos (Zapata y Castro, 1999). Mientras que Ayala et al. (2010) precisa que da lugar a tres tipos de transferencia de masa en contracorriente: transferencia de agua del producto a la solución osmótica, transferencia de soluto de la solución al producto y salida de solutos (azúcares, ácidos orgánicos, minerales y vitaminas) del producto hacia la solución, considerándose esta última como despreciable por ser cuantitativamente pequeña.

Sin embargo, Barbosa y Vega (2000), concluyeron que la pérdida de agua y la ganancia de sólidos por parte de la fruta aumentan a medida que aumenta la concentración de la solución osmótica y la temperatura, mientras que Machucay (2009) afirma que se debe reducir su tamaño del alimento para facilitar la velocidad de deshidratación osmótica y lograr las características deseadas.

El agente osmótico debe tener una alta solubilidad, un soluto de mayor peso molecular favorece la pérdida de agua en desmedro de la ganancia de sólidos, mientras que un soluto de bajo peso molecular favorece la ganancia de sólidos, siendo la sacarosa como el agente más usado, pudiéndose utilizar dextrosa y jarabe de maíz, dependiendo su uso del costo del soluto, compatibilidad con el producto y las características organolépticas del producto tratado (Torres et al., 2013). Pero Barman \& Badwaik,( 2017) indica que para la deshidratación osmótica (DO) de rodajas de carambola se puede utilizar glucosa, sacarosa, fructosa y glicerol.

Asimismo, la pérdida de agua y la velocidad de secado aumentan cuanto mayor sea la concentración de soluto de la solución osmótica, sin embargo, a concentraciones muy altas de soluto pueden causar que se forme una capa sobre la superficie de las frutas dificultando la pérdida de agua. (Baroni y Hubinger, 2000, citado por Garzón, 2014). La velocidad de transferencia de masa se incrementa con la temperatura y sobre $45^{\circ} \mathrm{C}$, empieza el pardeamiento enzimático y deterioro de aromas y sobre $60{ }^{\circ} \mathrm{C}$ se

Downloadable from: http://revistas.unu.edu.pe

Carretera Federico Basadre Km 6, Dirección de Producción Intelectual

Revista de Investigación Universitaria por Universidad Nacional de Ucayali se distribuye bajo una Licencia Creative Commons Atribución-NoComercial 4.0 Internacional. 
modifican las características de los tejidos, favoreciendo la ganancia de sólidos (Farkas y Lazar, 1969, citado por Garzón, 2014).

Por otro lado, Chuquillanqui (2017) estudio la cinética de deshidratado osmótico del kiwi verde, con soluciones osmóticas de sacarosa, miel y glucosa a concentraciones $(40,50$ y 60 ${ }^{\circ}$ Brix), siendo, para la ganancia de sólidos, el modelo de Peleg a $60{ }^{\circ}$ Brix obteniéndose un coeficiente de determinación para sacarosa $(\mathrm{R}$ $2=0,9949)$; para miel ( $\mathrm{R} 2=0,9972)$, para glucosa ( $\mathrm{R} 2=0,9967)$. Mientras que Yupanqui (2010) demostró que, para el mango criollo en rodajas, el jarabe de glucosa tiene mayor poder osmótico, obteniéndose un producto con una humedad de $58,14 \%$ y una concentración de sólidos solubles de $41^{\circ}$ Brix y el tiempo óptimo de deshidratado osmótico fue de 5 horas en el jarabe de sacarosa y 6 horas en el jarabe de glucosa.

En el presente trabajo se estudió la cinética de deshidratación osmótica, para definir los parámetros de madurez de la fruta, concentración de agente osmótico y temperatura de proceso para la obtención de carambolas osmodeshidratadas.

\section{Metodología}

Se recolectaron frutos con 7 a $10 \mathrm{~cm}$ de diámetro longitudinal y 5 a $7 \mathrm{~cm}$ de diámetro, y se analizó las características físico químicas, según lo indicado por la AOAC (2005), para los parámetros: $\mathrm{pH}$, acidez titulable, humedad, solidos solubles, cenizas, fibra cruda.

Para el proceso de deshidratación osmótica, se seleccionaron frutos en estado de maduración 2 y 3 , los cuales fueron lavados, luego fueron sumergidos en una solución de Hipoclorito de sodio al $0,5 \%$ por 15 minutos. Se retiraron las aristas, luego la cáscara dejando solamente la parte comestible, posteriormente sumergirlo en una solución de metabisulfito de sodio (200 ppm) por 10 minutos, con el fin de evitar el pardeamiento enzimático, luego se enjuagó. Se cortaron rodajas transversales en forma "estrellita", con un grosor de 5 a $7 \mathrm{~mm}$ y con peso entre 3,5 y 4,2 g, para facilitar la deshidratación, y se les quito las semillas manualmente. Se pesaron $300 \mathrm{~g}$ de fruta para cada tratamiento.

La miel de caña de azúcar, con $75^{\circ}$ Brix y un pH de 3,5, se diluyó con agua destilada, se pasteurizó a $50^{\circ} \mathrm{C}$, hasta llegar a concentraciones estudiadas $\left(40,50\right.$ y $60^{\circ}$ Brix).

Se sumergieron los trozos de carambola en las concentraciones de miel de caña de azúcar, en 
una relación de 1:10 (fruta: miel). La deshidratación osmótica se efectuó a 40 y 50 ${ }^{\circ} \mathrm{C}$ en forma constante durante todo el proceso osmótico, evaluándose la variación de peso, sólidos solubles y humedad, cada 15 min las dos primeras horas, cada 30 minutos las dos horas siguientes, y cada 1 hora las dos horas restantes.

Se retiraron los trozos de carambola de la miel de caña de azúcar y se les quitó el exceso de miel colocando en tamices de acero inoxidable, por un tiempo de cinco minutos, para luego ser colocadas en una estufa eléctrica, por cinco horas, a $85{ }^{\circ} \mathrm{C}$ para todos los tratamientos, para luego ser colocadas en desecadores para evitar la absorción de humedad, hasta su enfriamiento a temperatura ambiente, para ser colocados en envases de polipropileno con tapa para ser rotulados y almacenados a temperatura ambiente.

El análisis sensorial se realizó utilizando una escala hedónica de cinco puntos, calificándose: aroma, color, sabor y textura, y agrado general, para lo cual se contó con la participación de treinta panelistas semi entrenados.

Las pruebas microbiológicas, se desarrollaron mediante la N.T.C. No. 071-MINSA DIGESA, para determinar presencia de mohos y levaduras.

Para la evaluación de la cinética, se aplicó un Diseño de Bloque Completamente al azar (DBC) con arreglo factorial $2 \times 3 \times 2$ con tres repeticiones haciendo un total de 36 unidades experimentales. Asimismo, se aplicó la prueba de medias de Tukey al 0,05\% de significancia.

Tabla 1

Tratamientos estudiados

\begin{tabular}{cccc}
\hline Tratamiento & $\begin{array}{c}\text { Factor A: Estado } \\
\text { de maduración de } \\
\text { fruto }\end{array}$ & $\begin{array}{c}\text { Factor B: Concentraciones } \\
\text { de miel de caña de azúcar } \\
\left({ }^{\circ} \text { Brix }\right)\end{array}$ & $\begin{array}{c}\text { Factor C: } \\
\text { Temperatura } \\
\left({ }^{\circ} \mathbf{T}\right)\end{array}$ \\
\hline 1 & & 40 & 40 \\
2 & & & 50 \\
3 & Medio pintón & 50 & 40 \\
4 & & 60 & 50 \\
5 & & 40 & 40 \\
6 & Pintón & & 50 \\
\hline 7 & & & 40 \\
8 & & & 50 \\
\hline
\end{tabular}

634

Downloadable from: http://revistas.unu.edu.pe

Carretera Federico Basadre $\mathrm{Km}$ 6, Dirección de Producción Intelectual 


\begin{tabular}{ccc}
\hline 9 & 50 & 40 \\
10 & & 50 \\
11 & 60 & 40 \\
12 & & 50 \\
\hline
\end{tabular}

\section{Resultados y discusiones}

Las características fisicoquímicas de los frutos de carambola en los dos estados de maduración y osmodeshidratada se observan en la tabla 2, donde se puede observar las diferencias especificas en cuanto al tipo de estado de maduración de los frutos frescos de carambola, en cuanto a los contenidos de Acidez, pH, Brix, Cenizas y Fibra, los cuales fueron más altos en frutos pintón, observándose a su vez que la humedad fue mayor en el fruto medio pintón. La caracterización fisicoquímica de la carambola osmodeshidratada muestra una disminución en la acidez, y un aumento significativo en el pH, ${ }^{\circ}$ Brix, cenizas y fibra.

\section{Tabla 2}

Caracterización fisicoquímica de los frutos de carambola fresca y osmodeshidratada.

\begin{tabular}{lccc}
\hline \multirow{2}{*}{ Análisis fisicoquímico } & \multicolumn{2}{c}{ Estado de maduración de fruta } & \begin{tabular}{c} 
Carambola \\
deshidratada \\
\cline { 2 - 3 } osmóticamente
\end{tabular} \\
\hline & $2,31 \pm 0.44$ & $2,0 \pm 0,17$ & $0,10 \pm 0,01$ \\
Acidez $^{1}(\%)$ & $2,09 \pm 0,17$ & $2,45 \pm 0,21$ & $4,22 \pm 0,09$ \\
$\mathrm{pH}^{1}$ & $3,78 \pm 0,32$ & $5,03 \pm 0,42$ & \\
Brix $^{1}$ & $31,52 \pm 5,94$ & $33,75 \pm 2,49$ & $55,67 \pm 2,08$ \\
Humedad (\%) & $91,42 \pm 0,75$ & $88,86 \pm 0,88$ & $9,81 \pm 0,27$ \\
Cenizas (\%) & $0,92 \pm 0,12$ & $1,22 \pm 0,21$ & $7,09 \pm 0,81$ \\
Fibra $(\%)$ & $4,05 \pm 0,27$ & $5,12 \pm 0,22$ & $25,81 \pm 0,77$ \\
\hline
\end{tabular}

${ }^{1}$ Los valores corresponde a la media \pm SD, para $n=9$

${ }^{2}$ Los valores corresponde a la media $\pm \mathrm{SD}$, para $\mathrm{n}=3$

\section{Cinética de deshidratación osmótica}

Variación de peso
La pérdida de peso en las rodajas de carambola se incrementó en función a la concentración del agente osmótico y temperatura de proceso, 
siendo altamente significativa a $60^{\circ}$ Brix y $50^{\circ} \mathrm{C}(\mathrm{P} \leq 0,05)$ (figura 1 y 2$)$ en comparación con el resto de las concentraciones, esto indica que la naturaleza del agente osmótico es fundamental para definir el comportamiento del producto durante el proceso de deshidratación tal como lo menciona Barat (1998).

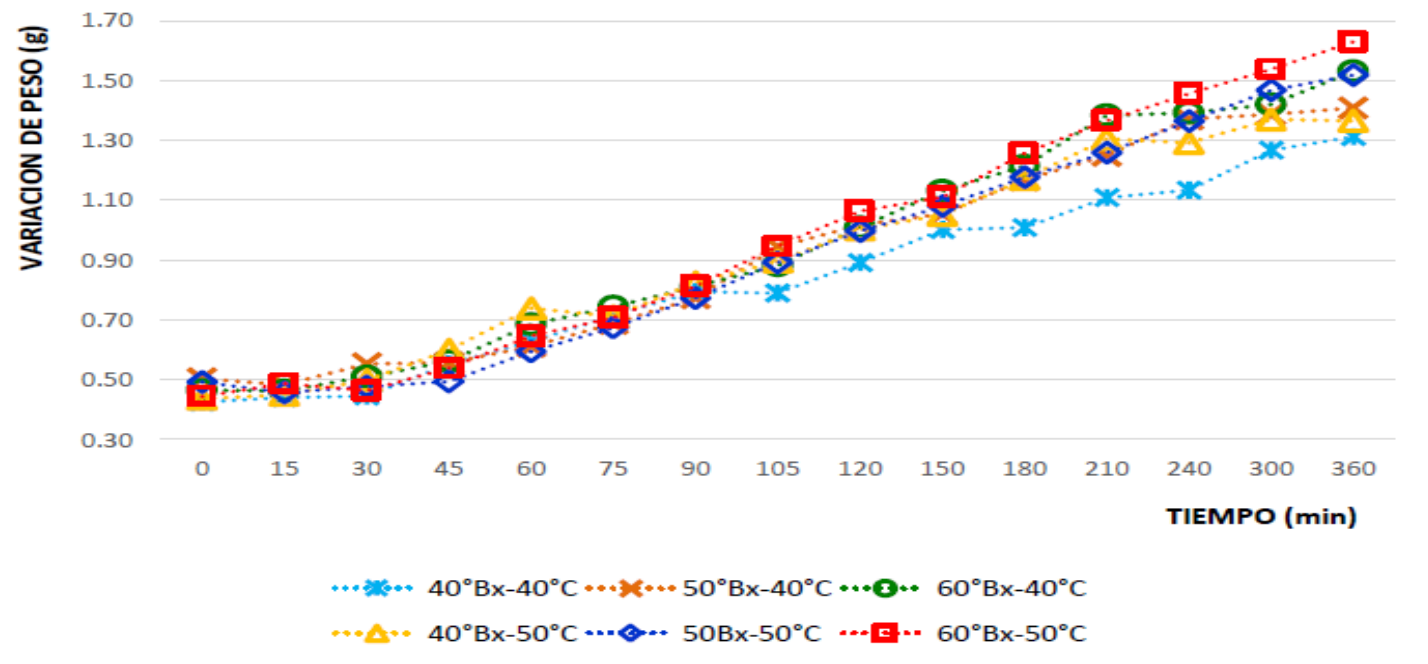

Figura 1: Variación de peso de la carambola pintón

A concentraciones de 50 y $60^{\circ}$ Brix de miel de caña de azúcar, la fruta llega a perder 1,33 $\mathrm{g}$ de agua para $50^{\circ}$ Brix y 1,51 g para $60^{\circ}$ Brix durante los 360 minutos.

De acuerdo a Maldonado, Santapaola, Singh y Garay (2008), la mayor pérdida de agua la alcanzó durante los primeros 60 minutos de proceso de las cuales un aumento de tiempo no produjo cambios apreciables en el contenido de agua de las muestras de yacón en condiciones de sacarosa a $40^{\circ}$ Brix lográndose a partir de los 90 minutos la estabilización del sistema, esto indica que la variación de la física natural de la fruta afecta al comportamiento osmótico y al estado final de los productos, pues no todos los alimentos presentarán el mismo comportamiento (Lenart y Flink, 1984). 


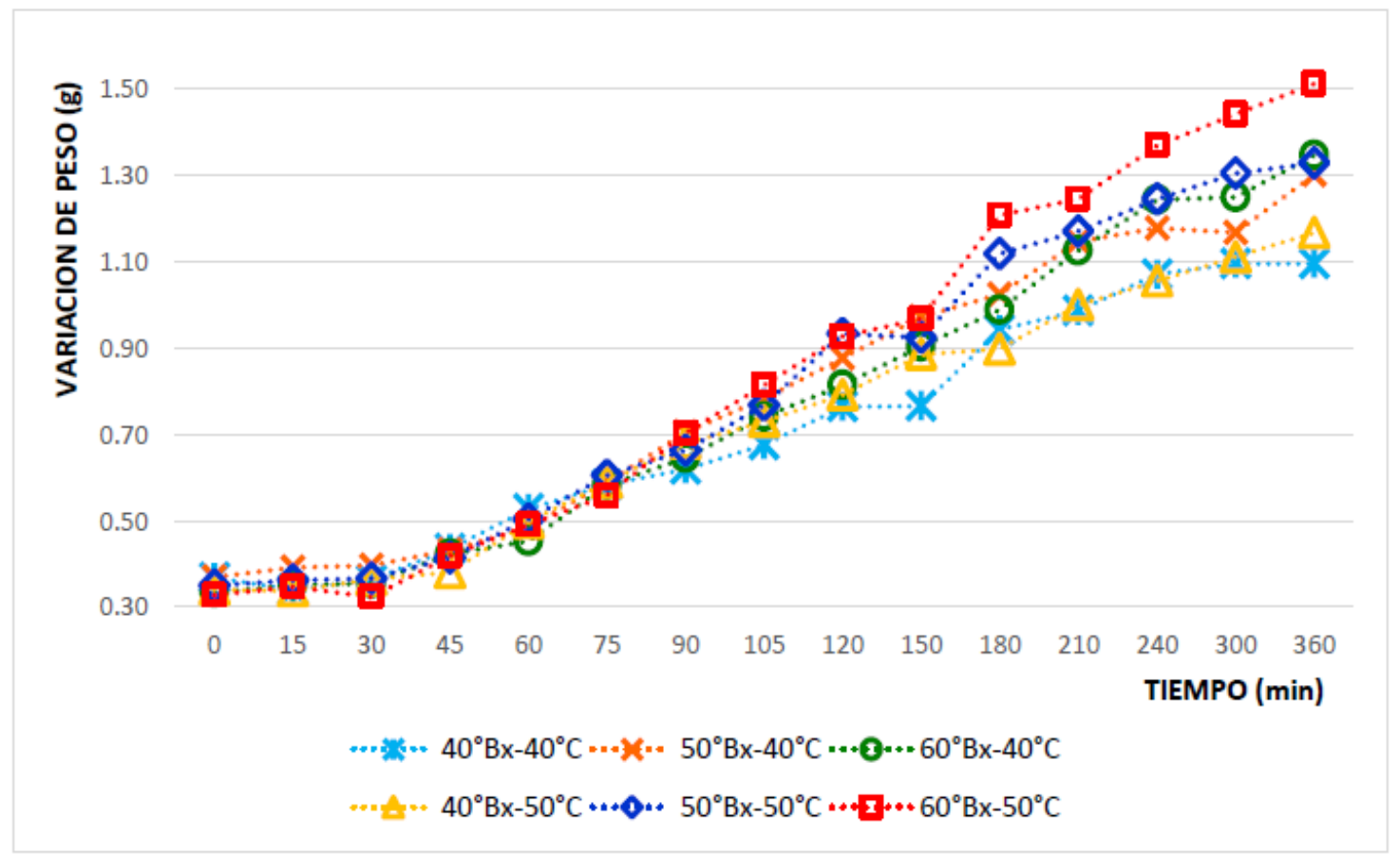

Figura 2: Variación de peso de la carambola medio pintón

Los contenidos de Acidez, $\mathrm{pH}$, Brix, Cenizas y Fibra, fueron más altos en frutos pintón, observándose a su vez que la humedad fue mayor en el fruto medio pintón, asimismo, la carambola osmodeshidratada muestra una disminución en la acidez, y un aumento significativo en el $\mathrm{pH},{ }^{\circ}$ Brix, cenizas y fibra. Por otro lado, la variación de peso de la carambola pintón y medio pintón, muestran que a concentraciones de 50 y $60^{\circ}$ Brix de miel de caña de azúcar, la fruta llega a perder 1,33 $\mathrm{g}$ de agua para $50^{\circ}$ Brix y $1,51 \mathrm{~g}$ para $60^{\circ}$ Brix durante los 360 minutos.

Contenido de sólidos solubles y humedad.
La tabla 3 muestra los promedios de los factores estudiados para el contenido de solidos solubles y humedad, donde se puede observar que en frutos pintones se produce la mayor ganancia de sólidos solubles, en comparación con los frutos medio pintones. Asimismo, la ganancia de sólidos solubles se incrementa al incrementarse la concentración de la solución osmótica empleada, el cual concuerda con Castillo y Cornejo (2007), deduciendo que a la mayor concentración se logra una mayor incorporación de sólidos. Finalmente, la ganancia de sólidos solubles es directamente proporcional a la temperatura de 
proceso, siendo menor cuando la temperatura es $40^{\circ} \mathrm{C}$, incrementándose a $50^{\circ} \mathrm{C}$, coincidiendo con Barrera (2011) el cual afirma que al elevar la temperatura se estimula el movimiento molecular y aumenta la permeabilidad celular.

Tabla 3

Contenido de solidos solubles y humedad, en los distintos factores de estudio

\begin{tabular}{|c|c|c|}
\hline \multicolumn{3}{|c|}{ Madures de la fruta } \\
\hline Factor A: Madurez & ${ }^{\circ} \mathrm{Brix}^{1}$ & Humedad $^{1}$ \\
\hline Medio Pintón & $29,55 \pm 0,09$ a & $79,77 \pm 0,09$ a \\
\hline Pintón & $30,04 \pm 0,09 \mathrm{~b}$ & $76,08 \pm 0,09 \mathrm{~b}$ \\
\hline \multicolumn{3}{|c|}{ Concentraciones de las soluciones osmóticas } \\
\hline Factor B: Concentración & ${ }^{\circ} \mathrm{Brix}^{1}$ & Humedad $^{1}$ \\
\hline $40^{\circ}$ Brix & $26,32 \pm 0,11$ a & $79,28 \pm 0,11 \mathrm{a}$ \\
\hline $50^{\circ}$ Brix & $29,87 \pm 0,11 \mathrm{~b}$ & $77,45 \pm 0,11 \mathrm{~b}$ \\
\hline $60^{\circ}$ Brix & $33,20 \pm 0,11 \mathrm{c}$ & $77,04 \pm 0,11 \mathrm{c}$ \\
\hline \multicolumn{3}{|c|}{ Temperatura de proceso } \\
\hline Factor C: Temperatura & ${ }^{\circ}$ Brix $^{1}$ & Humedad $^{1}$ \\
\hline $40^{\circ} \mathrm{C}$ & $29,11 \pm 0,09$ a & $78,37 \pm 0,09$ a \\
\hline $50^{\circ} \mathrm{C}$ & $30,48 \pm 0,09 \mathrm{~b}$ & $77,48 \pm 0,09 \mathrm{~b}$ \\
\hline
\end{tabular}

${ }^{1}$ Los valores representan la media \pm SD

Por lo tanto, la pérdida de humedad es mayor en frutos pintón, seguido del fruto medio pintón, lo que indica que a mayor porosidad mayor es la transferencia de masa (Fito, 1994), asimismo, a mayor concentración de solución osmótica, mayor es la perdida de humedad, sin embargo, un incremento en la concentración del agente osmótico puede provocar un aumento de la velocidad de transferencia de materia. Finalmente, a mayor temperatura la perdida de humedad es mayor, según Lazarides (1998), la difusión depende de la temperatura, por lo que a mayores temperaturas de proceso se promueve una mayor pérdida de agua debido a una mayor plasticidad de las membranas celulares.

Los frutos pintones muestran mayor ganancia de sólidos solubles, y la ganancia de sólidos solubles se incrementa al incrementarse la concentración de la solución osmótica empleada, y la ganancia de sólidos solubles es directamente proporcional a la temperatura de proceso, siendo menor cuando la temperatura es $40^{\circ} \mathrm{C}$, incrementándose a $50^{\circ} \mathrm{C}$. La pérdida 
de humedad es mayor en frutos pintón, asimismo, a mayor concentración de solución osmótica, mayor es la perdida de humedad, finalmente, a mayor temperatura la perdida de humedad es mayor.

Tabla 4

Relación cinética de la pérdida de agua y ganancia de sólidos por cada tratamiento en cada intervalo de tiempo

\begin{tabular}{|c|c|c|c|c|c|c|c|}
\hline \multirow{2}{*}{$\begin{array}{c}\text { Tratamiento } \\
\mathbf{S}\end{array}$} & \multicolumn{7}{|c|}{ Tiempos (minutos) } \\
\hline & 60 & 120 & 180 & 210 & 240 & 300 & 360 \\
\hline \multirow{3}{*}{$\mathrm{T} 1$} & & $1,03 \pm 0,0$ & & $1,40 \pm 0,2$ & $1,55 \pm 0,1$ & & $1,60 \pm 0,1$ \\
\hline & $0,53 \pm 0,08$ & 3 & $1,16 \pm 0,12$ & 9 & 4 & $1,65 \pm 0,14$ & 3 \\
\hline & & $1,06 \pm 0,0$ & & $1,57 \pm 0,0$ & $1,62 \pm 0,0$ & & $1,67 \pm 0,0$ \\
\hline \multirow[t]{2}{*}{$\mathrm{T} 2$} & $0,29 \pm 0,06$ & 7 & $1,44 \pm 0,11$ & 5 & 4 & $1,64 \pm 0,02$ & 3 \\
\hline & & $0,92 \pm 0,0$ & & $1,55 \pm 0,1$ & $1,59 \pm 0,1$ & & $1,73 \pm 0,0$ \\
\hline \multirow[t]{2}{*}{$\mathrm{T} 3$} & $0,49 \pm 0,03$ & 8 & $1,27 \pm 0,22$ & 9 & 4 & $1,66 \pm 0,08$ & 9 \\
\hline & & $1,30 \pm 0,1$ & & $1,81 \pm 0,0$ & $1,82 \pm 0,0$ & & $1,80 \pm 0,0$ \\
\hline \multirow[t]{2}{*}{$\mathrm{T} 4$} & $0,73 \pm 0,06$ & 6 & $1,62 \pm 0,16$ & 4 & 6 & $1,79 \pm 0,04$ & 7 \\
\hline & & $1,05 \pm 0,0$ & & $1,57 \pm 0,1$ & $1,65 \pm 0,1$ & & $1,81 \pm 0,1$ \\
\hline \multirow[t]{2}{*}{$\mathrm{T} 5$} & $0,33 \pm 0,04$ & 6 & $1,42 \pm 0,16$ & 4 & 1 & $1,75 \pm 0,15$ & 7 \\
\hline & & $1,00 \pm 0,1$ & & $1,45 \pm 0,1$ & $1,60 \pm 0,0$ & & $1,73 \pm 0,0$ \\
\hline \multirow{2}{*}{ T6 } & $0,45 \pm 0,11$ & 1 & $1,29 \pm 0,14$ & 4 & 9 & $1,68 \pm 0,06$ & 5 \\
\hline & & $0,87 \pm 0,1$ & & $1,31 \pm 0,0$ & $1,42 \pm 0,0$ & & $1,40 \pm 0,0$ \\
\hline \multirow[t]{2}{*}{$\mathrm{T} 7$} & $0,46 \pm 0,11$ & 3 & $1,10 \pm 0,07$ & 3 & 8 & $1,44 \pm 0,02$ & 4 \\
\hline & & $0,91 \pm 0,0$ & & $1,35 \pm 0,0$ & $1,47 \pm 0,0$ & & $1,51 \pm 0,0$ \\
\hline \multirow[t]{2}{*}{$\mathrm{T} 8$} & $0,25 \pm 0,07$ & 5 & $1,21 \pm 0,04$ & 5 & 2 & $1,47 \pm 0,03$ & 4 \\
\hline & & $0,82 \pm 0,0$ & & $1,34 \pm 0,0$ & $1,41 \pm 0,0$ & & $1,51 \pm 0,0$ \\
\hline \multirow[t]{2}{*}{ T9 } & $0,32 \pm 0,06$ & 9 & $1,12 \pm 0,11$ & 3 & 2 & $1,47 \pm 0,02$ & 3 \\
\hline & & $1,01 \pm 0,0$ & & $1,35 \pm 0,2$ & $1,42 \pm 0,0$ & & $1,51 \pm 0,0$ \\
\hline \multirow[t]{2}{*}{$\mathrm{T} 10$} & $0,35 \pm 0,18$ & 8 & $1,19 \pm 0,20$ & 0 & 2 & $1,47 \pm 0,06$ & 2 \\
\hline & & $1,03 \pm 0,0$ & & $1,53 \pm 0,0$ & $1,59 \pm 0,0$ & & $1,75 \pm 0,0$ \\
\hline \multirow[t]{2}{*}{$\mathrm{T} 11$} & $0,41 \pm 0,02$ & 4 & $1,41 \pm 0,08$ & 5 & 7 & $1,69 \pm 0,03$ & 8 \\
\hline & & $0,97 \pm 0,0$ & & $1,41 \pm 0,0$ & $1,58 \pm 0,0$ & & $1,72 \pm 0,0$ \\
\hline $\mathrm{T} 12$ & $0,32 \pm 0,03$ & 3 & $1,28 \pm 0,01$ & 6 & 8 & $1,63 \pm 0,04$ & 6 \\
\hline
\end{tabular}

Modelización de las ganancias de sólidos y pérdida de agua de carambolas osmodeshidratadas
La tabla 4 muestra la modelización de la ganancia de sólidos y perdida de agua de los tratamientos estudiados. Donde se puede observar que todos los tratamientos señalan efectividad de deshidratado osmótico. El 
tratamiento 12 (fruto pintón, con $50^{\circ} \mathrm{C}$ y $60^{\circ}$

Brix) es capaz de conseguir la mayor pérdida de agua, señalando una efectividad de 1,72 \pm 0,06 a partir de los 150 min de osmodeshidratado, siendo el tratamiento 7 (fruto pintón, $40^{\circ} \mathrm{C}$ y $40{ }^{\circ}$ Brix) el que inicio su efecto de perdida de agua a los 180 minutos de deshidratado, consiguiendo $1,40 \pm 0,04$ de eficiencia.

La eficiencia de la relación cinética señala cuando la pérdida de agua / Ganancia de sólidos es superior a 1.

Como se puede observar todos los tratamientos señalan efectividad de deshidratado osmótico. El tratamiento 12 (fruto pintón, con $50^{\circ} \mathrm{C}$ y $60^{\circ}$ Brix) es capaz de conseguir la mayor pérdida de agua, señalando una efectividad de 1,72 $\pm 0,06$ a partir de los 150 min de osmodeshidratado.

Los tratamientos estudiados no ejercen efecto significativo sobre el agrado general de la carambola osmodeshidratada

\section{Análisis sensorial y microbiológico}

La figura 3, muestra los resultados de la evaluación sensorial de la carambola osmodeshidratada para el agrado general, donde se observa que los tratamientos no ejercen efecto significativo $(P \geq 0,05)$ sobre el agrado general de la carambola osmodeshidratada. Esto implica que las carambolas sometidas a diferentes condiciones de deshidratación osmótica presentan similar característica con respecto a los atributos mencionados.

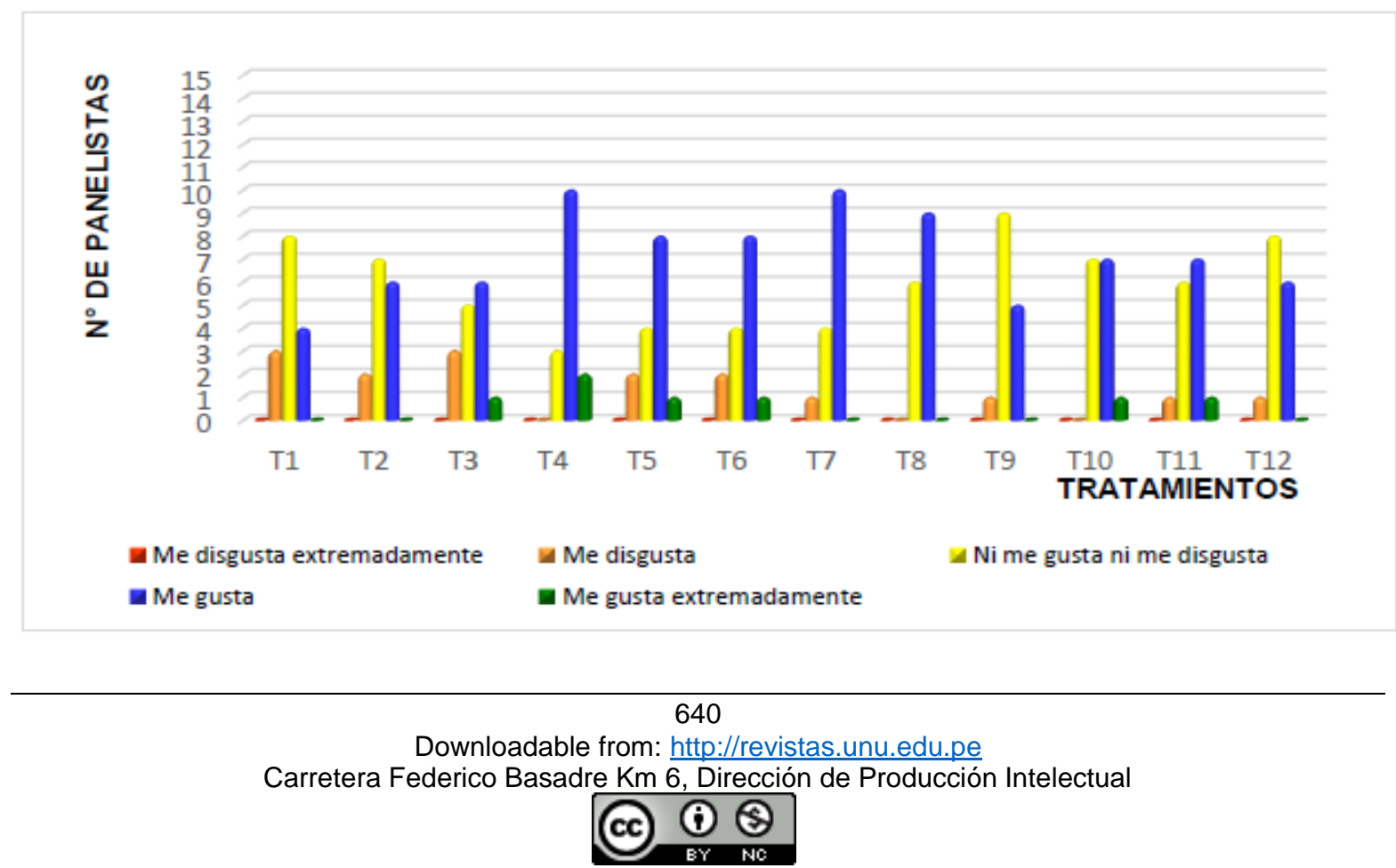

Revista de Investigación Universitaria por Universidad Nacional de Ucayali se distribuye bajo una Licencia Creative Commons Atribución-NoComercial 4.0 Internacional. 
Figura 3: Resultados de la evaluación sensorial de la carambola deshidratada osmóticamente para agrado general.

Por otro lado los resultados del análisis microbiológico realizado a las muestras de carambola osmodeshidratada, indican que para el recuento de mohos y levaduras, se observan un recuento inferior al 50ufc/g, es una cantidad aceptable según MINSA/DIGESA (2015) para productos deshidratados debido a la utilización de la solución de metabisulfito de sodio 200 ppm, indicándose además que la solución osmótica al disminuir sus ${ }^{\circ}$ Brix y con el transcurrir del tiempo tuvo una ligera tendencia a la fermentación esto se pudo notar por el aroma que se percibió después de desechar la solución osmótica.

\section{Conclusión}

El deshidrato de osmótico en la carambola con diferentes concentraciones de miel de caña de azúcar y temperaturas de fue adecuado, puesto que permitió obtener resultados favorables, para poder conservar y/o alargar el tiempo de vida útil, puesto que es una de las frutas con alto contenido de vitamina $\mathrm{c}$ y es recomendable su consumo también en otras presentaciones.

\section{Agradecimientos}

A la Universidad Nacional de Ucayali por el financiamiento del trabajo de investigación.

\section{Referencia bibliográfica}

A.O.A.C. (2005). Official methods of Analysis. (18.a ed.).

Ayala, A., Giraldo, C., Serna, L. (2009). Efecto de la agitación sobre la deshidratación osmótica de pitaya amarilla (Selenicereus megalanthus S.). Revista Científicas de América Latina y el Caribe, España y Portugal.

Barat, M. (1998). Desarrollo de un modelo de la deshidratación osmótica como operación básica. España: Universidad Politécnica DE Valencia.

Barbosa, C., G; Vega, M., H. (2000). Deshidratación de alimentos. Zaragoza, España. Editorial Acribia. 297 p.

Barman, N., \& Badwaik, L. S. (2017). Effect of ultrasound and centrifugal force on carambola (Averrhoa carambola L.) slices during osmotic dehydration. Ultrasonics Sonochemistry, 34, 37-44. https://doi.org/10.1016/j.ultsonch.2016. 05.014 
Barrera, C. (2001). Influencia de la estructura en la transferencia de masa durante la deshidratación osmótica y aplicación de la impregnación a vacío en el confitado de piña. España: Universidad Politécnica de Valencia.

Castillo, M. y Cornejo, F. (2007). Estudio del efecto del proceso de deshidratación osmótica en la obtención de trozos secos de carambola. Revista tecnológica ESPOL, volumen 20 pp; 183188.

Castillo-Zamudio, R., Cabal Prieto, A., Vidaña-Reyes, $\quad$ T., $\quad$ \& Salgado_Cervantes, M. (2018). Uso conbinado de técnologías de secado asistidas por ultrasonidos de potencia para la conservación de carambola ( Averroha carambola L .). 3(February 2018), 270-276.

Chuquillanqui Romero, L. M. 2017. Modelamiento de Azuara, Magee y Peleg en cinética de deshidratación osmótica de Kiwi. Tesis para optar el título de Ingeniera en Industrias alimentarias. Facultad de Ingeniería en Industrias Alimentarias. Universidad Nacional del Centro del Perú. Huancayo, Perú. Disponible en: http://repositorio.uncp.edu.pe/bitstream
/handle/UNCP/4117/Chuquillanqui\%2

0Romero.pdf?sequence=1\&isAllowed $=\mathrm{y}$

Fito, P. (1994). Modelling of vacuum osmotic dehydration of food. Journal of Food Engineering. $\quad 22: \quad 313-328$. https://doi.org/10.1016/02608774(94)90037-X

Garzón, M. (2014). Bombones rellenos de mashua (Tropaeolum tuberosum) deshidratadas por ósmosis. (Tesis para la obtención del título ingeniería de alimentos, facultad de ciencias de la ingeniería). universidad tecnología equinoccial, Ecuador

Lazarides, N. (1998). Mass transfer phenomena during osmotic processing of fruits and vegetables. Drying. Leuven, October 23-25, 4345. https://doi.org/10.1111/j.13652621.1994.tb02095.x

Machucay, S. (2009). Deshidratación osmótica de frutas. (Tesis para la obtención grado maestría). Universidad Nacional del Centro del Perú. Junín, Perú.

Meneses, J. (2018). Osmoconvective dehydration in fruits and vegetables: A review of recent developments. 
Agroindustrial Science, 8(1), 67-72.

https://doi.org/10.17268/agroind.sci.201

8.01 .10

MINSA/DIGESA. (2015). Norma sanitaria que establece los criterios microbiológicos de calidad sanitaria e inocuidad para los alimentos y bebidas de consumo humano. V-01.

Ochoa Icaza, K. K. (2019). Calidad sensorial de cuatro cruces experimentales de cacao (Theobroma cacao L.) adicionando niveles de pasta de frutas deshidratadas carambola (Averrhoa carambola) y coco (Cocos nucifera) para la obtención de chocolate negro. Universidad Técnica Estatal de Quevedo.

Pucahuaylla, E. R., \& Valdivieso, marlhiz N. (2018). Efecto Del Osmodeshidratado Y Secado Por Aire Caliente Sobre La Capacidad Antioxidante, B-Carotenos, Cinética Y Rehidratación En El

Liofilizado De Carambola. Universidad Nacional del Centro del Perú. de Ingeniero Alimentos). Universidad Nacional de Trujillo, Perú.

Yupanqui Cristóbal, E. A. 2010. Influencia de la sacarosa y glucosa en la deshidratación osmótica del mango criollo (Manguifera indica $\quad$ L.) de Satipo. Tesis para optar el título de Ingeniera en Ciencias Agrarias, especialidad de Industrias Alimentarias.

Facultad de Ciencias Agrarias. Universidad Nacional del Centro del Perú. Satipo, Perú. Disponible en: http://repositorio.uncp.edu.pe/bitstream /handle/UNCP/1892/Yupanqui\%20Cris tobal.pdf?sequence $=1 \&$ is Allowed $=y$

Zapata, E. y Castro, G. (1999). Deshidratación osmótica de frutas y vegetales. Revista Facultad Nacional de Agronomía, Medellín. Vol. 52, No.1; p. 451-466.

Downloadable from: http://revistas.unu.edu.pe Carretera Federico Basadre Km 6, Dirección de Producción Intelectual 